\title{
Stabilisation of refraction following cataract surgery
}

\author{
PETER R BARANYOVITS \\ From Wolverhampton Eye Infirmary, Comptom Road, Wolverhampton
}

SUMmaRY Refraction was performed at frequent, regular intervals for six months following routine intracapsular cataract extraction. Patients were divided into two groups, those whose limbal sections were closed with 8-0 virgin silk or with 9-0 nylon. The stabilisation of refraction was observed, and the most suitable time to prescribe 'first glasses' was estimated retrospectively. In the silk group this was found to be at three months in the nylon group at four months. However, results in the latter were less predictable with greater variation.

This report was undertaken to determine the rate of stabilisation of refraction following intracapsular cataract extraction, with the aim of improving efficiency of the 'first glasses' prescription.

Many authorities recommend prescribing glasses when serial refraction shows no significant change. If this procedure is followed carefully, it may require frequent clinic visits. Hence first glasses are often prescribed after a fixed postoperative period of about two months. This has been the practice for several decades, during which surgical practices and patient expectations have changed greatly.

Glasses prescribed too early, before refraction has stabilised, may cause disappointment and unnecessary expense for the patient; too late prescription may prolong disability and frustration.

Changes in corneal curvature following cataract surgery have been recognised for over a century, having been first described by Donders' in 1864 . Many other workers have since investigated the nature of postcataract astigmatism. The effects of the following factors have been studied: type, ${ }^{2-4}$ depth, ${ }^{5}$ and number $r^{b}$ of sutures, pre- or postplaced sutures, ${ }^{6}$ wound placement, ${ }^{7}$ and size of wound ${ }^{8}$ Change of corneal curvature as a function of time has also been considered.

In 1935 Groenholm ${ }^{4}$ reported keratometric studies on 200 eyes following cataract extraction without sutures and with one or two sutures. The initial high degree of astigmatism at 10 days was found to be halved at two months. Particular attention was paid to resultant astigmatism and frequent regular keratometry was not included.

Floyd " reported on a total of 47 eyes following

Correspondence to Dr P R Baranyovitis, The Lawns, 19 Chetwynd Aston, Newport, Shropshire TF10 9LJ. cataract surgery with Graefe section and two sutures. Keratometry was performed at monthly intervals and he found 'significant' changes in corneal curvature, up to three months after surgery. He suggested that glasses prescribed before this time may be of a temporary nature. More recently Reading ${ }^{8}$ reported on 43 eyes undergoing intracapsular cataract extraction sutured with virgin silk. These patients were followed up at monthly intervals for one year by photokeratometry. Changes in corneal curvation after one month were found to be 'small'.

The present study, conducted at Wolverhampton Eye Infirmary, was designed to help resolve the following questions: How quickly does refraction stabilise sufficiently to allow prescription of satisfactory glasses? Is this rate consistent in different patients? Is it influenced by the type of suture and the initial degree of corneal distortion produced during surgery?

\section{Material and methods}

\section{PATIENTS}

From the beginning of the study all patients undergoing uncomplicated cataract extraction were included provided they were able, co-operative, and had no other serious ocular disease. A total of 43 patients, 47 eyes (average age 70 years, range 49-86 years, $95 \%$ confidence interval $2 \cdot 8$ ) were followed up for six months.

\section{SURGERY}

Five different surgeons performed the operations using a similar microsurgical technique: ab externa stepped incision (approx $160^{\circ}$ ) followed by one peripheral iridectomy and irrigation with $\alpha$ - 
chymotrypsin. Intracapsular cataract extraction was then performed with a cryoprobe. The wounds were closed either by interrupted 8-0 virgin silk (5-7 sutures) or by a double 9-0 nylon bootlace (each one closing half the wound, giving a total of six bites). The choice of suture alternated between patients; 24 eyes were closed with virgin silk, 23 eyes with nylon. A total of 15 eyes had iris clip intraocular lenses inserted (Binkhorst or Boberg-Ans types), nine in the virgin silk group and six in the nylon group. All patients were prescribed a topical steroid (betamethasone $0 \cdot 1 \%$ ), four times a day for four weeks; this was then reduced and discontinued over the following two weeks.

\section{FOLLOW-UP}

All patients were followed up by the author for six months. Keratometry was performed preoperatively and in the first week following surgery along with the first refraction. Refraction was repeated at two-weekly intervals for three months, thereafter monthly for three further months.

Keratometry was performed on a Bausch-Lomb keratometer. All refraction was carried out with the same equipment, ensuring constant back vertex distance. Retinoscopy was followed by subjective refraction, the sphere confirmed by the duochrome test and the cylinder and axis by using Jackson's cross cylinder.

Analysis of the data was by either independent or paired $t$ test with $95 \%$ confidence intervals and $\chi^{2}$ tests. Pearson product moment correlations were also computed to investigate the relationship between certain variables.

\section{Results}

To display best the speed at which refraction stabilised after intracapsular cataract surgery the rate of change of each component, namely sphere, cylinder, and axis, was calculated from each patient's serial refractions. The units of change were dioptres/ week and degrees/week. The mean values are shown in Fig. 1 for the two suture groups, 8-0 virgin silk and 9-0 nylon.

Virgin silk sutures tend to lose their apparent strength in the second month after surgery. This shows itself by a sudden change in cylinder from with the rule to against the rule." In this study, six months after operation, significantly more of the silk group had a cylinder against the rule, $22(92 \%)$, as opposed to $4(17 \%)$ of the nylon group, $\left(\chi^{2}=23 \cdot 3, p<0 \cdot 001\right)$.

Fig. 1C shows this dramatic change taking place in the first two months (that is, a peak in the rate of change of axis), but thereafter the rate of change remained low. Both suture groups showed significant changes in all three components of refraction for at least the first three months.

In each graph the nylon suture curve shows a more gradual change which is also slightly more prolonged. The different shapes of the curves for the two suture groups are reflected in the differing 'prescribing times'. This was taken to be the time, following surgery, that the refraction was judged to be stable enough to allow prescription of satisfactory glasses. For the purposes of this study the prescribing time was estimated retrospectively when all the refraction results for the full six months were reviewed. The
Fig. 1 Comparison of the rate of change of each component of refraction in the two suture groups of interrupted 8-0 virgin silk and continuous 9-0 nylon, against time following intracapsular extraction.

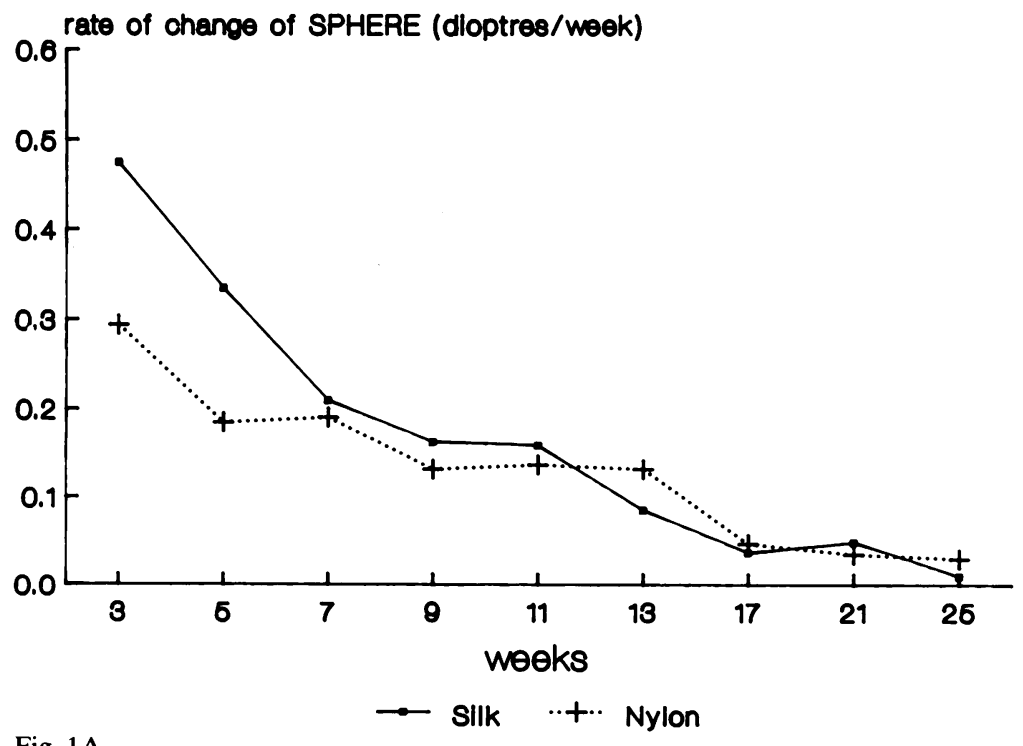

Fig. 1A 


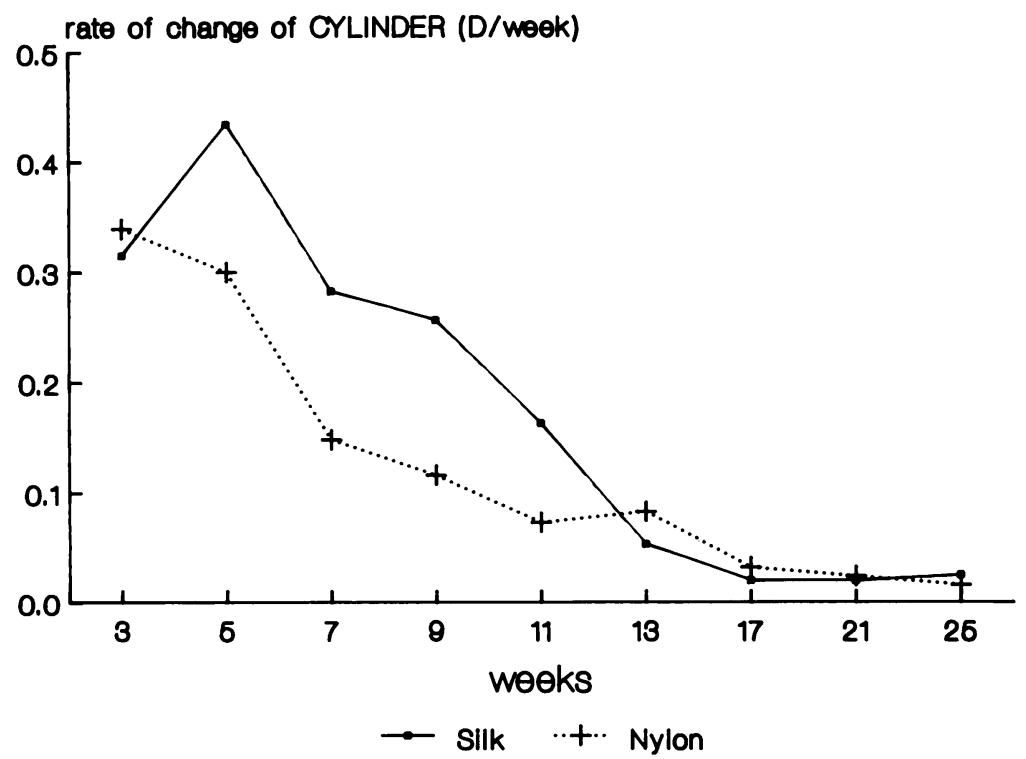

Fig. 1B

prescribing time was taken to be the earliest time that refraction approached the 'final' six-month result. The criteria used was a maximum difference of 0.5 dioptres in sphere, $0.25 \mathrm{D}$ in cylinder, and $5^{\circ}$ in axis.

The results are displayed in Fig. 2, which shows when the patients reached a stable refraction.

Statistical comparison of the mean prescribing time showed a significant difference between the two suture groups, $\left(t_{45}=2 \cdot 18, \mathrm{p}<0 \cdot 05\right.$; mean difference $=$ $2 \cdot 3$ weeks) (Table 1 ).
In each suture group there was no significant difference in prescribing times of eyes with and without intraocular lenses. Silk group mean difference with and without $\mathrm{IOL}=0.089$ weeks $(95 \%$ $\mathrm{CI} \pm 2 \cdot 05)$; nylon group mean difference $=2.45$ weeks (95\% CI $\pm 4 \cdot 21)$.

Pre- and postoperative keratometry was performed to estimate the degree of corneal distortion caused by the incision and sutures. The maximum change occurred about the vertical axis. This change,

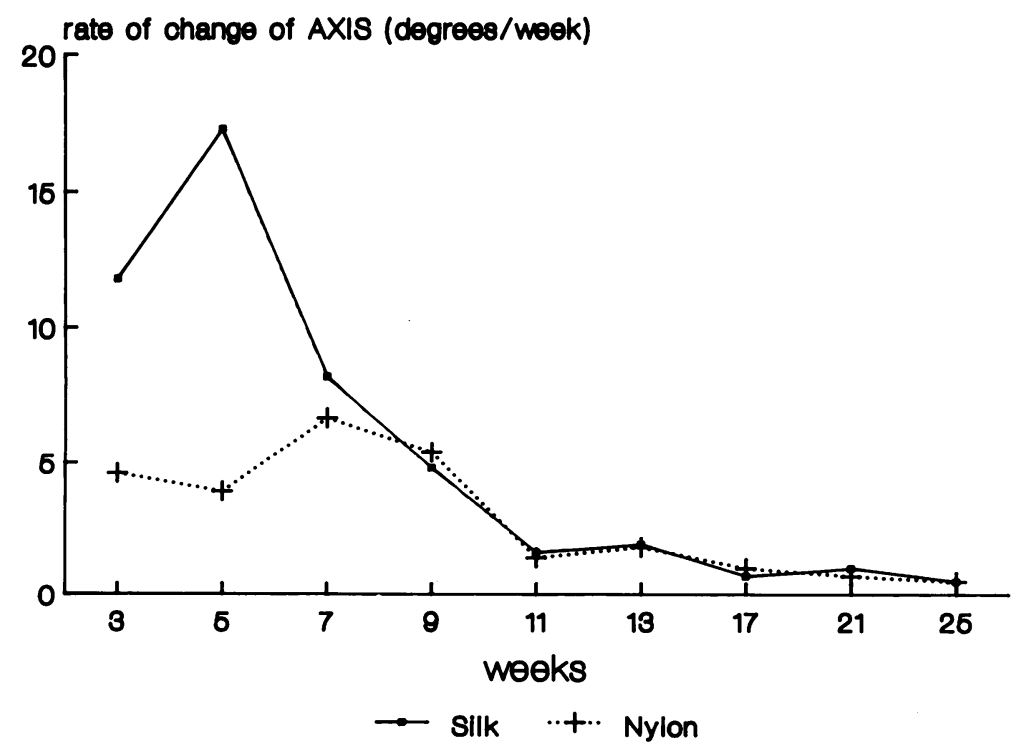

Fig. 1C 


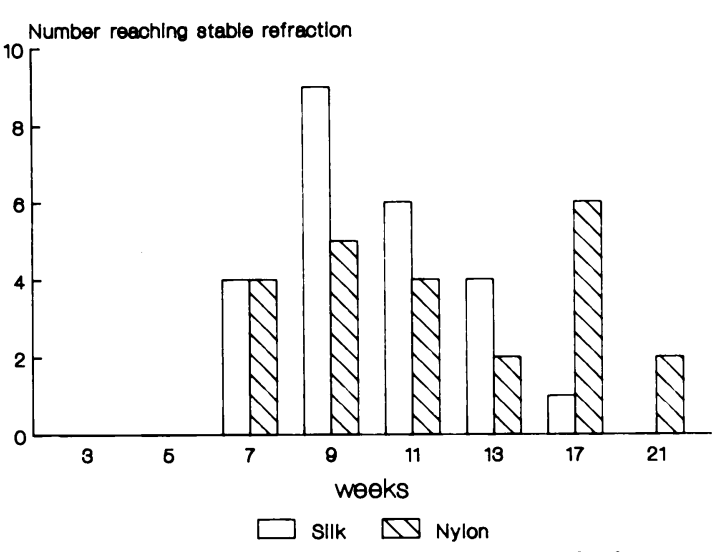

Fig. 2 Comparison of the rate of stabilisation of refraction in the two suture groups of interrupted 8-0 virgin silk and continuous 9-0 nylon, as measured by the time first glasses could be prescribed following intracapsular extraction.

measured in dioptres, was used as an estimate of surgically induced corneal distortion. This is shown in Table 2 with the initial (1st week) and final (6th month) cylinder and axis for comparison. Correlation analysis was performed to investigate their relationship (Table 3 ).

\section{Discussion}

The two different suture techniques, for intracapsular cataract extraction, examined in this study (interrupted 8-0 virgin silk and continuous 9-0 nylon) appear to have different effects on the course of postoperative refraction and its rate of stabilisation. Once stable, $22(92 \%)$ of the silk group have a cylinder (mean 2.13 D) against the rule, and 19 $(83 \%)$ of the nylon group remain with a cylinder (mean $1.58 \mathrm{D}$ ) with the rule.

As can be seen from Table 3 , in the silk group there was no significant relationship between the initial

Table 1 Comparison of first glasses 'prescribing time', following intracapsular extraction, between wounds closed with interrupted 8-0 virgin silk and continuous 9-0 nylon sutures

\begin{tabular}{|c|c|c|}
\hline & \multicolumn{2}{|l|}{ Suture material } \\
\hline & 8-0 Silk & 9-0 Nylon \\
\hline No. of eyes & 24 & 23 \\
\hline $\begin{array}{l}\text { Mean prescribing time } \\
\text { (weeks after surgery) }\end{array}$ & $10 \cdot 2$ & $12 \cdot 5$ \\
\hline $95 \% \mathrm{CI}$ & $\begin{array}{l} \pm 1 \cdot 0 \\
(9 \cdot 2-11 \cdot 2 \text { weeks })\end{array}$ & $\begin{array}{l} \pm 1.9 \\
(10 \cdot 6-14.4 \text { weeks })\end{array}$ \\
\hline
\end{tabular}

$\mathrm{CI}=$ confidence interval
Table 2 Comparison of surgical corneal distortion and initial and final astigmatism following intracapsular extraction sutured with interrupted 8-0 virgin silk or 9-0 continuous nylon

\begin{tabular}{|c|c|c|}
\hline & \multicolumn{2}{|c|}{ Suture material } \\
\hline & 8-0 Silk & 9-0 Nylon \\
\hline No. of eyes & 24 & 23 \\
\hline Mean corneal distortion & $\begin{array}{r}2.75 \mathrm{D} \\
( \pm 0 \cdot 66)^{*}\end{array}$ & $\begin{array}{c}3.36 \mathrm{D}) \\
( \pm 0.74)\end{array}$ \\
\hline Mean initial cylinder, 1st week & $\begin{array}{l}1.84 \mathrm{D} \\
( \pm 0.41)\end{array}$ & $\begin{array}{l}2.69 \mathrm{D} \\
( \pm 1 \cdot 1)\end{array}$ \\
\hline Mean final cylinder, 6th month & $\begin{array}{l}2 \cdot 13 \mathrm{D} \\
( \pm 0 \cdot 39)\end{array}$ & $\begin{aligned} & 1.58 \mathrm{D} \\
&( \pm 0.38)\end{aligned}$ \\
\hline Mean initial axis & $\begin{array}{l}1(0) \cdot 4^{\circ} \\
( \pm 10 \cdot 5)\end{array}$ & $\begin{array}{c}98.9^{\circ} \\
( \pm 6.6)\end{array}$ \\
\hline Mean final axis & $\begin{array}{l}174 \cdot 4^{\circ} \\
( \pm 16 \cdot 5)\end{array}$ & $\begin{array}{l}117^{\circ} \\
( \pm 2())\end{array}$ \\
\hline Mean prescribing time, 1 st glasses & $\begin{array}{l}10 \cdot 2 \text { weeks } \\
( \pm 1 \cdot 0)\end{array}$ & $\begin{array}{l}12.5 \text { weeks } \\
( \pm 1.9)\end{array}$ \\
\hline
\end{tabular}

*95\% confidence interval in parentheses.

corneal distortion and the time that the postoperative refraction was considered stable enough to prescribe first glasses ('prescribing time'). The initial corneal distortion is dependent on the positioning and tension of the sutures. But as silk loses its apparent strength in four to six weeks, ${ }^{12}$ the initial distortion can be expected to have little correlation with the prescribing time and the final degree of cylinder. The loss of tension in the silk suture results in a dramatic change in cylinder, as shown in Fig. 1.

In the nylon group the relationship between initial corneal distortion and prescribing time was significant, that is, eyes with most distortion take longest to stabilise. Nylon sutures lose their tensile strength very gradually and cause less adjacent tissue reaction. Hence the initial distortion caused by the suture will influence the behaviour of the wound for a longer period of time than silk. The limbal tissue adjacent to the suture may undergo a certain degree of moulding, and the suture itself may cheese-wire through the tissue slightly. These effects would allow a reduction and equalisation of the tension across the wound. Hence one may expect the amount of

Table 3 Correlation analysis of the relationship between the degree of initial surgical corneal distortion, final cylinder. and 'prescribing time', in the two suture groups of interrupted 8-0 virgin silk and continuous 9-0 nylon

\begin{tabular}{lll}
\hline & \multicolumn{2}{l}{ Suture material } \\
\cline { 2 - 3 } & $8-0$ Silk & $9-0$ Nylon \\
\hline Initial distortion $v$ prescribing time & $r_{22}=0 \cdot() 2$ & $r_{21}=0.41$ \\
Initial distortion $v$ final cylinder & $r_{22}=0 \cdot 11$ & $r_{21}=0 \cdot 12$ \\
Final cylinder $v$ prescribing time & $r_{22}=0.39$ & $\left.r_{21}=-0\right) \cdot 21$ \\
\hline
\end{tabular}


cylinder to lessen before the wound has stabilised fully. This appears to be the case in the nylon group, as the mean initial cylinder of $2.69 \mathrm{D}$ (axis $98.9^{\circ}$ ) reduces to $1.58 \mathrm{D}\left(\right.$ axis $117^{\circ}$ ) at six months. A similar result was found by Wyman, ${ }^{3}$ who followed up 100 cases sutured with 9-0 and 10-0 nylon.

The relationship between final cylinder and prescribing time shows an interesting difference between the two suture groups. It suggests that the eyes with longer prescribing times have wounds which take longer to reach sufficient strength to stabilise refraction. Before reaching this point the refraction can be influenced by the changing tensions or effective loss of sutures. In the silk group the longer prescribing times are associated with a larger final cylinder $(92 \%$ against the rule), that is, more time for stretching of the wound before it reaches sufficient strength to become stable. In the nylon group the longer prescribing times are associated with a smaller final cylinder ( $83 \%$ with the rule), that is, more time for stretching of the sutures and moulding of adjacent limbal tissue. In this study there was no significant relationship in either suture group between the initial corneal distortion and the amount of final cylinder.

Of particular interest was the rate at which refraction stabilised sufficiently to allow prescription of first glasses. Here there was a significant difference between the nylon and silk suture groups. The mean prescribing time in the silk group was $10 \cdot 2$ weeks $(95 \% \mathrm{CI} \pm 1.0)$; in the nylon suture group not only was the mean prescribing time later at 12.5 weeks $(95 \% \mathrm{CI} \pm 1.9)$ but also the spread was greater.

In absolute terms only $13(54 \%)$ of the silk suture group were considered sufficiently stable to prescribe first glasses at the traditional postoperative period of two months. This reached $23(96 \%)$ at three months and $24(100 \%)$ at four months. In the nylon suture group nine $(39 \%)$ were stable at two months, 15 $(65 \%)$ at three months, $21(91 \%)$ at four months, and $23(100 \%)$ at five months.

In summary, three months would appear to be a practical time to refract and order glasses for patients whose eyes have been sutured with virgin silk. This should improve efficiency and avoid multiple refractions in the great majority of patients. However, when an eye has been sutured with continuous nylon, the stabilisation is less predictable and more caution is required. It may be reasonable to wait four months before ordering first glasses. However, after the third month the changes were fairly predictable: $87 \%$ showed a slight drop in the amount of cylinder of up to 0.75 dioptres, with only small changes in axis. Hence, if it is necessary to prescribe glasses earlier, a slightly smaller cylinder than indicated could be given.

Limbal wounds closed by sutures or techniques different from above, ${ }^{+11}$ such as interrupted 10-0 nylon, may well respond differently, and the smaller sections used in extracapsular cataract surgery, particularly phacoemulsification, can be expected to have less effect on postoperative refraction. However, it is unlikely that the large sections of intracapsular surgery, if closed by a different technique, would stabilise any quicker, as the speed of wound healing, which is a major factor, would remain largely unchanged.

The help of Dr M F Collins, of the North Staffordshire Hospital Centre, over the statistical analysis is gratefully acknowledged.

\section{References}

1 Donders FC. On the anomalies of accommodation and refraction of the eye. London: New Sydenham Society, 1864: 334.

2 Pearce JL. Discussion on suture material. Adv Ophthalmol 1970; 22: $58-9$.

3 Wyman GJ. Comparison of results: 100 cases with 8-0 silk versus 100 cases with 9-0-10-() nylon. In: Emery JM, Paton D, eds. Current concepts in cataract surgery. St Louis: Mosby, 1974: 91-2.

4 Stainer GA, Binder PS, Parker WT, Perl T. The natural and modified course of post-cataract astigmatism. Ophthalmic Surg 1982; 13: 822-7.

5 Van Rij G, Waring GO. Changes in corneal curvature induced by sutures and incisions. Am J Ophthalmol 1984; 98: 773-83.

6 Singh D, Kumar K. Kerametric changes after cataract extraction. Br J Ophthalmol 1976: 60: 638-41.

7 Thrasher BH, Boemer CF. Control of astigmatism by wound placement. J Am Intraocul Implant Soc 1984; 10: 176-80.

8 Reading VM. Astigmatism following cataract surgery. $\mathrm{Br} J$ Ophthalmol 1984; 68: 97-104.

9 Groenholm V. Corneal suture of cataract incision in relation to postoperative astigmatism. Finska Läkaresällskapets Handlingar. 1935; $78: 286$.

10 Floyd $\mathrm{G}$. Changes in the corneal curvature following cataract extraction. Am J Ophthalmol 1951: 34: 1525-33.

11 Gills JP. The effect of cataract sutures on postoperative astigmatism. Am J Optom Physiol Opt 1974: 51: 97-100.

12 Elenius V. Karo T. Changes in the refractive power of the cornea after cataract extraction. Eye, Ear, Nose and Throat Monthly 1968; 47: 54-60.

Accepted for publication 3 September 1987. 